\title{
Comparação de Alguns Modelos Matemáticos para Ajuste à Curva de Lactação Média de um Rebanho da Raça Caracu ${ }^{1}$
}

\author{
Lenira El Faro², Lucia Galvão de Albuquerque ${ }^{3,4}$, Luiz Alberto Fries ${ }^{3,5}$
}

\begin{abstract}
RESUMO - No presente estudo foi estimada a curva de lactação média de 7239 lactações de vacas da raça Caracu, com base em 299.949 controles semanais. As funções ajustadas aos dados médios da curva de lactação foram Linear Hiperbólica e Quadrática Logarítmica, como lineares, e Gama Incompleta e Polinomial Inversa, como não-lineares. Os parâmetros foram estimados por meio de processos iterativos, método de Gauss-Newton. O Coeficiente de Determinação ajustado $\left(\mathrm{R}_{A}^{2}\right)$, o Teste de Durbin-Watson (DW) e os desvios $(\%)$ entre as produções totais (PTL) observadas e estimadas foram usados como medida da qualidade do ajuste. Os resíduos estimados, apesar de pequenos, apresentaram correlação serial positiva, pois o teste DW foi significativo para as quatro funções estudadas, indicando violação da pressuposição de independência entre estes resíduos. Os $\mathrm{R}_{A}^{2}$ foram superiores a 97\% e os desvios entre as PTL observadas e estimadas, mínimos, indicando que, de acordo com estes critérios, qualquer função poderia ser usada para ajustar a curva de lactação média desse rebanho. Quanto aos componentes da curva, a melhor aproximação foi obtida pela função Polinomial Inversa, para produção inicial, tempo e produção no pico. As funções Gama Incompleta e Quadrática Logarítmica apresentaram ajustes semelhantes, porém a primeira apresentou melhor definição dos parâmetros e componentes da curva de lactação.
\end{abstract}

Palavras-chave: curva de lactação, persistência, produção de leite

\section{Comparison of some Mathematical Models for the Adjustment of the Average Milking Curve of Caracu Breed Herd}

\begin{abstract}
The present study was carried out to estimate the average lactation curve of 7239 lactations of Caracu cows based on 299,949 weekly records. The functions fitted to the mean data of the lactation curve were: Linear Hyperbolic and Logarithmic Quadratic as linear and Incomplete Gamma and Inverse Polynomial function as non-linear. The parameters were estimated by iterative methods, GaussNewton method. The adjusted Coefficient of Determination $\left(\mathbf{R}_{A}^{2}\right)$, the Durbin-Watson test (DW) and the deviations (\%) from actual and predicted total milk yield were used as measurement of goodness of fit. The estimated residues, even though small, presented a positive serially correlation because the DW test was significant for the four studied functions, indicating violation of the assumption of the independence among the residues. The $\mathrm{R}_{A}^{2}$ were higher than $97 \%$, and the deviations between actual and predicted milk yields were small, indicating that, in accordance with this criterion, any function could be used to fit the average lactation curve of the herd. Regarding to the components of the lactation curve the best approach was obtained by the Inverse Polynomial function for initial production, time and production on peak. The Incomplete Gamma and Logarithmic Quadratic functions presented similar adjustment, however, the first one presented better definition of parameters and components of the lactation curve.
\end{abstract}

Key Words: lactation curve, persistency, milk production

\section{Introdução}

A curva de lactação pode ser definida como a representação gráfica da produção de leite em todo o período da lactação. A utilização de funções matemáticas para a descrição de curvas de lactação tem dois objetivos principais: um é o estudo da forma da curva e suas implicações na produção de leite, o que auxiliaria na determinação de curvas desejáveis no sentido econômico ou biológico, de acordo com o objetivo de seleção para um rebanho ou para grupos de animais. Conhecendo a forma da curva de lactação de uma vaca, por exemplo, um criador poderia usá-la como informação adicional, além da produção total, para tomar decisões de descarte ou de manejo. A decisão seria baseada em um desvio entre a curva "padrão", desejável sob o ponto de vista econômico e biológico, e a curva estimada para cada indivíduo, por

\footnotetext{
${ }^{1}$ Parte da dissertação de Mestrado apresentada à FCAV-UNESP, Campus de Jaboticabal, financiada pelo CNPq.

${ }_{2}^{2}$ Pesquisador Científico-Instituto de Zootecnia-SP.

${ }^{3}$ Professor da FCAV-UNESP, Campus de Jaboticabal-SP.

${ }^{4}$ Bolsista do CNPq.

${ }^{5}$ Bolsista da FAPESP.

${ }^{6}$ Gensys Consultores Associados - Porto Alegre - RS.
} 
intermédio de uma função matemática. Outro objetivo do uso de funções matemáticas para o ajuste de curvas de lactação é a estimação da produção total de leite, partindo-se de registros incompletos. Isso implicaria em economia de tempo e redução dos custos, uma vez que um animal poderia ser avaliado antes de ter sua lactação terminada, ou no caso de touros jovens, antes que suas filhas tivessem suas lactações encerradas.

As funções matemáticas usadas para o ajuste às curvas de lactação podem ser classificadas, em relação aos seus parâmetros, como lineares, intrinsecamente lineares e não-lineares (AFIFI e CLARK, 1984). As lineares apresentam facilidades sobre as demais, pois seus parâmetros apresentam apenas relações aditivas entre si e podem ser estimados por regressões lineares, enquanto as intrinsecamente lineares necessitam de processos iterativos ou de transformações nos seus parâmetros. As funções não-lineares não sofrem transformações nos parâmetros e, portanto, são estimadas apenas por processos iterativos, uma vez que não se consegue isolar cada um dos elementos do vetor dos parâmetros para obtenção das soluções. Quando se tratam de curvas de actação, as funções intrinsecamente lineares são as mais comuns e, geralmente, seus parâmetros são estimados por meio de regressões lineares, após linearização; as mais conhecidas são a função Gama Incompleta (WOOD, 1967) e a função Polinomial Inversa (NELDER, 1966).

O objetivo do presente estudo foi comparar a qualidade do ajuste em relação à curva de lactação média e seus componentes, proporcionada por quatro funções matemáticas propostas na literatura, utilizando registros de produção de leite de um rebanho da raça Caracu.

\section{Material e Métodos}

As informações utilizadas no presente estudo foram obtidas do rebanho da raça Caracu da Fazenda Recreio, localizada em Poços de Caldas, MG. Quanto aos locais de ordenha, a propriedade é dividida em 14 retiros, dos quais dois são maternidade. A ordenha é manual, realizada duas vezes ao dia, com a presença do bezerro e o leite de um quarto de úbere por ordenha é reservado à sua alimentação, durante toda a lactação, quando então ocorre o desmame. O manejo reprodutivo utiliza período de monta, que se estende de meados de abril a meados de fevereiro do ano seguinte, e as novilhas são acasaladas quando atingem dois anos de idade e peso corporal entre 300 a $400 \mathrm{~kg}$. As informações utilizadas neste estudo são referentes ao período de 1978 a 1988, no total de 7239 lactações com controles semanais. Foram mantidas as lactações com, no mínimo, 13 controles, que foi considerado o período suficiente para estimar o ponto de máximo da curva. O número máximo de controles por lactação foi de 45 e, assim, as lactações foram truncadas aos 315 dias. Dessa maneira, o arquivo de dados utilizado para estimar a curva média apresentou total de 299.949 registros semanais.

As funções utilizadas para o ajuste à curva de lactação média desse rebanho foram:

Função Linear Hiperbólica (FLH)

$$
Y_{X}=a_{0}+a_{1} X+a_{2} X^{-1}
$$

\section{BIANCHINI SOBRINHO (1984)}

Função Quadrática Logarítmica (FQL)

$$
Y_{X}=a_{0}+a_{1} X+a_{2} X^{2}+a_{3} \ln (X)
$$

\section{BIANCHINI SOBRINHO (1984)}

Função Gama Incompleta (FGI)

$$
\begin{gathered}
Y_{X}=a_{0} X^{a_{1}} e^{-a_{2} X} \\
\text { WOOD (1967) }
\end{gathered}
$$

Função Polinomial Inversa (FPI)

$$
\begin{gathered}
Y_{X}=X\left(a_{0}+a_{1} X+a_{2} X^{2}\right)^{-1} \\
\text { NELDER (1966) }
\end{gathered}
$$

A variável dependente, YX, representa a produção de leite na semana $X$, e $a_{0}, a_{1}, a_{2}$ e $a_{3}$ são os parâmetros que compõem cada modelo. A estimação dos parâmetros de cada função foi realizada por meio de regressões não-lineares, usando-se o Método Modificado de Gauss-Newton, disponível no Proc Nlin (S AS Institute Inc., 1992). Foram feitas análises sobre a média dos controles, no total de 45 pontos a serem explicados e, também, sobre todos os 299.949 controles, incluindo, dessa maneira, todas as lactações.

As funções foram comparadas utilizando-se os seguintes critérios:

- Coeficiente de determinação ajustado ao número de parâmetros $\left(R_{A}^{2}\right)$ - DRAPER e SMITH, (1981);

- Teste ou estatística de Durbin-Watson DURBIN e WATSON (1951); e

- Desvios (\%) entre as produções totais de leite observadas e estimadas.

As produções totais estimadas por intermédio das funções de regressão (PTLE) foram representadas por: 


$$
P T L E=\sum_{X=1}^{D_{L}} F(\beta, X)
$$

em que

DL = duração da lactação; e

$\mathrm{F}(\beta, X)=$ cada uma das funções ajustadas.

As produções observadas (PTL) foram calculadas usando-se o Método Escada Corrigido, como utilizado por BIANCHINI SOBRINHO (1988).

Assim, os desvios foram estimados por:

$$
\operatorname{DESVIO}(\%)=\frac{(P T L-P T L E)}{P T L} .100
$$

\section{Resultados e Discussão}

\section{Curva de lactação média observada}

A curva de lactação média observada para esse rebanho Caracu está representada na Figura 1. A produção inicial de leite foi de $9,7 \mathrm{~kg}$. O pico de produção ocorreu na segunda semana de lactação, com 10,6 kg de leite, e a produção total foi de 1920,3 kg. De maneira geral, a curva média para esse rebanho assemelhou-se à curva de lactação de vacas zebuínas (MADALENA et al., 1979; BIANCHINI SOBRINHO, 1984; e GONÇALVES, 1994), que iniciam a lactação praticamente no pico e, após, há tendência de queda contínua na produção diária, determinando baixas persistências.

A curva média deste rebanho apresentou pequena mudança na curvatura, aproximadamente entre a $26^{\mathrm{a}}$ e a $36^{\mathrm{a}}$ semana de lactação, que fez com que a taxa de declínio na produção semanal variasse. Para esse rebanho, a ordenha é realizada com bezerro ao pé e um quarto de úbere é reservado à sua alimentação. No decorrer da lactação, espera-se que o desenvolvimento do bezerro faça com que ele procure, com maior freqüência, alimentos fibrosos e concentrados, fazendo com que a necessidade do seu aleitamento torne menor no decorrer da lactação. Isso poderia explicar a ocorrência dessa área crítica entre a $26^{\mathrm{a}} \mathrm{e}$ a 36a semana de lactação. Além da menor dependência por parte do bezerro, o manejo do rebanho também pode contribuir para esse fato, uma vez que as vacas com baixos níveis de produção de leite são secas antes do término da lactação e, por conseguinte, apenas as vacas mais produtivas tendem a permanecer na ordenha.

\section{Curvas de lactação estimadas}

Os resíduos estimados como a diferença entre as

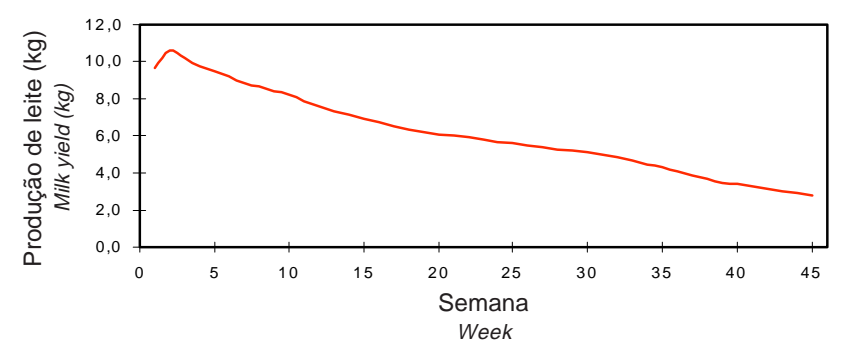

$\mathrm{Pl}=9,7 \mathrm{~kg} P \mathrm{P}=10,6 \mathrm{~kg} \quad \mathrm{TP}=2 \mathrm{sem} . \mathrm{PTL}=1920,3 \mathrm{~kg}$

Figura 1- Curva de lactação média observada para vacas Caracu.

Figure 1 - Observed average lactation curve of Caracu cows.

produções de leite observadas e as estimadas pelos modelos de regressão estão apresentados na Figura 2. Observa-se por meio da dispersão dos resíduos, os estádios em que as produções foram subestimadas ou superestimadas. As quatro funções estimaram resíduos pequenos por todo o período de lactação. As funções Quadrática Logarítmica e a Gama Incompleta apresentaram tendências idênticas, quanto à dispersão dos resíduos. Constatou-se essa mesma tendência também para a função Linear Hiperbólica até aproximadamente a 39a semana, embora os seus resíduos tenham sido maiores. A função Polinomial Inversa apresentou tendência diferente das observadas para as demais funções, pois estimou resíduos cujos valores foram mínimos até a 23a semana, indicando boa aproximação das produções estimadas em relação às observadas, mas, a partir daí, foram sempre maiores, até o fim da lactação. Observou-se, em relação às curvas delineadas pela distribuição dos resíduos, que houve tendência de autocorrelação serial positiva, uma vez que os mesmos se mostraram dispersos em blocos de valores positivos e negativos, alternando-se como sugerido por DRAPER e SMITH (1981).

As funções dos parâmetros, como produção inicial, tempo de pico e produção no pico, auxiliam na determinação da forma da curva de lactação, além de terem aplicações práticas. O problema de várias funções descritas na literatura está relacionado ao fato de que nem todas descrevem estas funções dos parâmetros com clareza. A função Gama Incompleta, por exemplo, descreve a produção inicial como função do intercepto $\left(\mathrm{a}_{0}\right)$. Este parâmetro, de acordo com COBBY e LE DU (1978), define uma escala de produção, e não a produção inicial. A persistência é o período pelo qual a lactação se mantém com altos níveis de produção de leite. Entretanto, para a função, essa medida não tem unidade, o que dificulta sua 
interpretação biológica. Sabe-se apenas que, quanto maior os seus valores absolutos, mais persistente é a lactação, mas quais valores seriam esperados ou possíveis é difícil decidir. As funções Linear Hiperbólica e Quadrática Logarítmica descrevem a persistência da lactação como taxa de declínio da produção semanal de leite, representada pelo coeficiente de regressão linear da reta $\left(a_{1}\right)$. Isso pode ser bem estimado por um polinômio ordinário de primeiro grau, que é composto por apenas dois parâmetros. Assim, o declínio da produção semanal seria medido em relação ao máximo, que para esse polinômio seria a produção inicial. Para funções contendo mais que dois parâmetros, como a Linear Hiperbólica e Quadrática Logarítmica, que contêm, respectivamente, 3 e 4 parâmetros, a estimação e a interpretação da persistência como taxa de declínio ficam mais complexas, uma vez que haveria coeficientes de regressão quadráticos e, ainda, para a Quadrática Logarítmica, um quarto coeficiente de regressão, que seria função do logaritmo de X. Para a função Polinomial Inversa, não há definição, na literatura, de fórmula para o cálculo da persistência da lactação, apenas para tempo e produção no pico.

Constam da Tabela 1 as equações, os parâmetros e as funções dos parâmetros estimados de acordo com cada função. As produções iniciais estimadas foram próximas da observada para todas as funções. Com exceção da função Polinomial inversa, as demais funções não estimaram pico de lactação ou tenderam a estimá-lo antes do parto. Este fato poderia ser esperado, uma vez que o mesmo ocorreu na segunda semana de lactação. Entretanto, estimativas de tempo ou produção no pico negativas não seriam biologicamente aceitáveis, apesar de poderem ocor- rer matematicamente, como estimado pela Quadrática Logarítmica. Isto pode se tornar um problema quando se trabalha com ajustes de lactações individuais em conjuntos de dados extensos, com raças zebuínas ou cruzadas, em que o aparecimento de curvas atípicas é grande e esperado.

A função Polinomial Inversa, apesar de descrever a produção inicial, o tempo e a produção no pico próximos do observado, apresentou resíduos maiores na segunda metade da lactação (Figura 2) e seu desvio em relação às produções totais de leite observadas e estimadas foi maior que para as demais funções.

A função Linear Hiperbólica difere de um polinômio ordinário de primeiro grau, por apresentar um parâmetro adicional $\left(a_{2}\right)$ relacionado ao inverso do tempo de lactação $(X)$, cuja principal importância está na descrição da fase de ascensão da lactação e do pico de produção. Como o parâmetro $a_{2}$ é diluído no decorrer da lactação, seu valor deve ser alto e negativo, para que se consiga estimar o ponto de máximo da curva. PINEDA (1987) obteve estimativas para este parâmetro, quando ajustou a curva de lactação de vacas Holandesas, variando de $-24,95$ a $-28,18 \mathrm{~kg}$, enquanto BIANCHINI SOBRINHO (1984), com lactações de vacas Gir, obteve estimativas variando de $-4,32$ a $-35,90 \mathrm{~kg}$, entre primeiras e terceiras lactações. No presente estudo, $a_{2}$ foi positivo e a função não estimou pico de produção, além de ter apresentado os maiores desvios no início da lactação, embora sua aproximação da PTL tenha sido melhor que para as demais funções.

Os testes estatísticos são apresentados na Tabela 2. Quando se trata do ajuste de curvas de lactação, os testes mais comumente usados na literatura para determinação da qualidade do ajuste são o coeficiente de

Tabela 1 - Equação média, produção inicial (PI), tempo de pico (TP), produção no pico (PP), produção total estimada (PTLE), e desvios para vacas Caracu, de acordo com as diferentes funções matemáticas

Table 1 - Mean equation, initial production (PI), time of peak (TP), peak production (PP), total milk yield (PTLE) and deviations estimated according to the different mathematical functions

\begin{tabular}{|c|c|c|c|c|c|c|c|}
\hline $\begin{array}{l}\text { Função } \\
\text { Function } \\
\end{array}$ & & $\begin{array}{l}\mathrm{PI}^{1} \\
(\mathrm{~kg})\end{array}$ & $\begin{array}{c}\mathrm{TP}^{2} \\
\text { (semanas) }\end{array}$ & $\begin{array}{l}\mathrm{PP}^{3} \\
(\mathrm{~kg})\end{array}$ & $\begin{array}{c}\mathrm{S}^{4} \\
(\mathrm{~kg})\end{array}$ & $\begin{array}{l}\text { PTLE }^{5} \\
(\mathrm{~kg})\end{array}$ & $\begin{array}{l}\text { Desvios } \\
\text { kge }(\%)\end{array}$ \\
\hline$Y=9,599-0,157 X+1,048(X)^{-1}$ & $\begin{array}{l}\text { Linear hiperbólica } \\
\text { Linear hyperbolic }\end{array}$ & 10,491 & - & - & $-0,157$ & 1917,41 & $2,86(0,15)$ \\
\hline$Y=10,654-0,205 X+0,0013 X^{2}-0,246 \ln (X)$ & $\begin{array}{l}\text { Quadrática logarítmica } \\
\text { Logarithmic quadratic }\end{array}$ & 10,450 & $-1,18$ & - & $-0,205$ & 1925,59 & $5,32(-0,28)$ \\
\hline $\mathrm{Y}=\mathrm{X}\left(0,021+0,077 \mathrm{X}+0,0044 \mathrm{X}^{2}\right)^{-1}$ & $\begin{array}{l}\text { Polinomial inversa } \\
\text { Inverse polynomial }\end{array}$ & 9,692 & 2,21 & 10,567 & - & 1939,38 & $19,11(-1,00)$ \\
\hline $\mathrm{Y}=10,587 \mathrm{X}^{0,0079} \mathrm{e}^{-0,0274 X}$ & $\begin{array}{l}\text { Gama incompleta } \\
\text { Incomplete gamma }\end{array}$ & 10,301 & 0,30 & 10,338 & 3,626 & 1927,60 & $7,33(-0,38)$ \\
\hline
\end{tabular}

${ }_{1}^{1}$ Prod. inicial; ${ }^{2}$ Tempo de pico; ${ }^{3}$ Produção no pico; ${ }^{4}$ Persistência; ${ }^{5}$ Produção total estimada; ${ }^{6}$ Desvio \%=[(1920,27-PTLE)/1920,27]x100

${ }^{1}$ Initial production; ${ }^{2}$ Time of peak; ${ }^{3}$ Peak production; ${ }^{4}$ Persistency; ${ }^{5}$ Total milk yield; ${ }^{6}$ Deviation \%=[(1,920.27-PTLE)/1,920.27]x100 

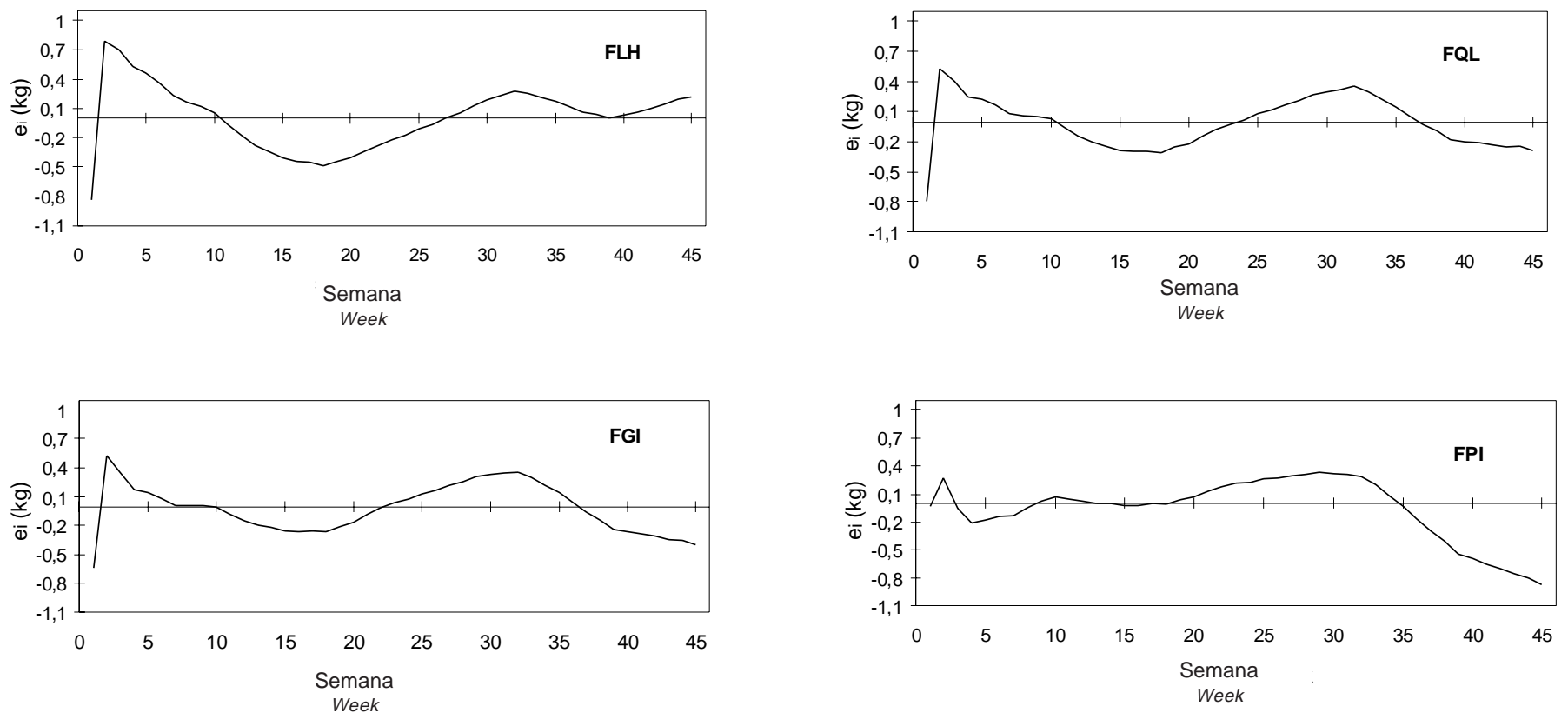

Figura 2 - Gráfico de dispersão dos resíduos estimados $\left(\mathrm{e}_{\mathrm{i}}\right)$ para a curva de lactação média de vacas Caracu, de acordo com as quatro funções matemáticas.

Figure 2 - Residuals estimated $\left(e_{j}\right)$ for the average lactation curve, according to the four mathematical functions of Caracu cows.

Tabela 2 - Coeficiente de determinação ajustado $\left(\mathrm{R}_{\mathrm{A}}^{2}\right)$, teste de Durbin-Watson ( $D W$, realizados para a curva de lactação média de vacas Caracu, de acordo com cada função matemática

Table 2 - Adjusted coefficient of determination $\left(\mathrm{R}_{\mathrm{A}}^{2}\right)$, DurbinWatson statistic (DW), estimates for the average lactation curve of Caracu cows, according to mathematical functions

\begin{tabular}{lcc}
\hline Função & $\mathrm{R}_{\mathrm{A}}^{2}(\%)$ & $\mathrm{DW} *$ \\
Function & 97,66 & 0,610 \\
Linear hiperbólica & $(42,90)^{* *}$ & \\
Linear hyperbolic & 98,56 & 0,656 \\
Quadrática logarítmica & $(43,40)$ & \\
Logarithmic quadratic & 98,60 & 0,550 \\
Gama incompleta & $(43,50)$ & \\
Incomplete gamma & 97,62 & 0,078 \\
Polinomial inversa & $(43,30)$ & \\
Inverse polynomial &
\end{tabular}

* Referência para DW significativo $\left(D_{L}<1,29\right)$.

** Regressão feita sobre todos os controles (299.949).

* DW significant when $D_{L}<1.29$.

** Regression based on total controls $(299,949)$. determinação ajustado $\mathrm{R}_{\mathrm{A}}^{2}$ e o teste de Durbin-Watson. De acordo com BIANCHINI SOBRINHO (1984), bons ajustes seriam obtidos para $\mathrm{R}_{\mathrm{A}}^{2}$ iguais ou superiores a $80 \%$. No presente estudo, a curva média foi estimada por intermédio de regressões sobre todos os controles e também sobre a média dos controles, como é usual. Foram estimados $\mathrm{R}_{\mathrm{A}}^{2}$ elevados para as quatro funções ajustadas, indicando que qualquer uma poderia ser utilizada no ajuste da curva de lactação média desse rebanho. O teste de DW, entretanto, foi significativo para todos os modelos ajustados, indicando correlação serial positiva entre os resíduos estimados, já constatada no gráfico de dispersão dos resíduos (Figura 2). Dessa maneira, a pressuposição feita em relação aos erros, de independência, foi violada e os modelos seriam considerados incorretos ou ineficazes (HOFFMAN e VIEIRA, 1987). Esta 
forma de correlação pode ocorrer em curvas de lactação, porque as observações estão ordenadas em função do tempo e, de certa forma, alguns autores consideram esperada a presença de correlação residual, pois os resíduos contêm toda variação nãoexplicada pelo modelo, relativas aos efeitos genéticos e de ambiente permanente, uma vez que são desvios de uma mesma vaca. Apesar de esperada, a correlação residual em análises de regressão pode gerar alguns problemas, pois os parâmetros estimados passam a ter interpretações diferentes (GLASBEY, 1988). De acordo com esse autor, isso ocorre porque, como o conjunto de dados é explicado pelos parâmetros que compõem o modelo de regressão e pelo erro, mudanças ocorrendo nos erros forçariam mudanças compensatórias nesses parâmetros.

De maneira geral, não há consenso na literatura de quais testes estatísticos devem ser considerados na avaliação da aderência proporcionada por uma função matemática. Testes estatísticos como $\mathrm{R}_{\mathrm{A}}^{2}$, por exemplo, dão idéia de como é a aproximação de $\hat{Y}$ em relação a $Y$, mas pouco dizem da aproximação biológica da curva de lactação, principalmente com relação à sua forma. Por outro lado, como tomar decisões com relação à adequação de uma função, quando testes estatísticos como DW indicaram quebra das pressuposições do método de quadrados mínimos, mesmo havendo boas aproximações em relação à forma da curva e seus componentes, ou elevados coeficientes de determinação, como foi o caso da função Polinomial Inversa?

\section{Conclusões}

De acordo com os coeficientes de determinação ajustados, qualquer função poderia ser utilizada para explicar a curva de lactação média deste rebanho. Entretanto, todos os modelos de regressão falharam, considerando-se que os resíduos foram correlacionados.

A função Polinomial Inversa poderia descrever componentes da curva de lactação como produção inicial e tempo e produção no pico, uma vez que estes são descritos no início da lactação e esta função apresentou ajuste superior às demais até a $21^{\mathrm{a}}$ semana de lactação. Esta função, entretanto, não descreve o principal componente econômico da curva, que é a persistência da lactação.
As funções Gama Incompleta e Quadrática Logarítmica apresentaram resultados semelhantes, quando houve ajuste da curva média desse rebanho, mas a primeira apresenta melhores definições para parâmetros e funções dos parâmetros.

\section{Referências Bibliográficas}

AFIFI, A.A., CLARK, V. 1984. Computer aided multivariate analysis. Belmont, California: Lifetime Learning. 458p.

BIANCHINI SOBRINHO, E. Estudo da curva de lactação de vacas da raça Gir. Ribeirão Preto, SP: USP, 1984. 88p. Tese (Doutorado em Genética) - Universidade de São Paulo.

BIANCHINI SOBRINHO, E. Estimativa da produção total de vacas da raça Gir, baseada em controles semanais, quinzenais, mensais e bimestrais. Obtenção de fatores multiplicativos. Ribeirão Preto, SP: USP, 1988. 90p. Tese (Livre Docência) - Universidade de São Paulo.

COBBY, J.M., LE DU, L.P. 1978. On fitting curves to lactation data. Anim. Prod., 26:127-133.

DRAPER, N.M., SMITH, H. 1981. Applied regression analysis, 2.ed. New York: John Wiley. 407p.

DURBIN, J., WATSON, G.S. 1951. Testing for serial correlation in least squares regression. Biometrika, 37:409-428.

GLASBEY, C.A. 1988. Examples of regression with serially correlated errors. Statistician, 37:277-291.

GONÇALVES, T.M. Estudo da curva de lactação de vacas da raça Gir. Viçosa, MG: UFV, 1994. 66p. Dissertação (Mestrado em Zootecnia) - Universidade Federal de Viçosa.

HOFFMANN, R., VIEIRA, S.O. 1987. Análises de regressão: econometria, 2.ed. São Paulo: Hucitec. 339p.

MADALENA, F.E., MARTINEZ, M.L., FREITAS, A.F. 1979. Lactation curves of Holstein-Friesian and Holstein-Friesian x Gir cows. Anim. Prod., 29:101-107.

NELDER, J.A. 1966. Inverse polynomials a useful group of multifactor response functions. Biometrics, 22:128-141.

PINEDA, A.M.G. Mathematical functions applied to dairy cow lactation curves. 1987. 243p. Thesis (Master of Science), University of Florida.

SAS User's Guide: Statistics. SAS Institute Inc., Cary, North Caroline, 1992. 842p.

WOOD, P.D.P. 1967. Algebraic model of the lactation curve in cattle. Nature, 216:164-165.
Recebido em: 06/10/97

Aceito em: 16/04/99 